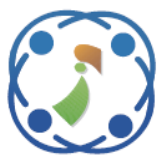

\title{
Contribution in Enhancing the Remaining Useful Life Prediction in Abrupt Failures: Bearing Case
}

\author{
Tahar Boukra $^{1 *} \quad$ Yacine Bensafia $^{2} \quad$ Khatir Khettab $^{3}$ \\ ${ }^{1}$ Université 20 Août 1955 Skikda, Laboratoire Electrotechnique Skikda-LES, Algeria \\ ${ }^{2}$ Université de Bouira, bouira 10000, Algeria \\ ${ }^{3}$ Mohamed Boudiaf University of M'sila 28000, Algeria \\ * Corresponding author's Email: t.boukra@univ-skikda.dz
}

\begin{abstract}
For a simple and accurate prediction of the Remaining Useful Life (RUL) of a component/system, degradation-based algorithms, deployed by data-driven prognostic model, attempt to track a sensed or preprocessed feature called prognostic feature, highly correlated with fault growth. This feature should reflect the fault evolution through the entire component/system life, i.e. having a monotonic trend shape. Extracted features usually show undesirable behaviors such as fluctuation, non-monotonicity and abrupt increase at the end of the component lifecycle which hampers the accurate prediction of the RUL. We must, therefore, be addressed to the identification of new prognostic features having an obvious monotonic trend shape to enhance the prediction of the RUL. In this context, this paper attempts to address this issue by further preprocessing the extracted features in a way that the identified prognostic feature results in a smoothed and trended shape. The qualities of the identified feature are evaluated by a set of established and proposed suitability metrics. Datasets from bearings run-to-failure experiments provided by FEMTO-ST Institute - IEEE PHM 2012 challenge- were used to validate our approach. A mean percentage error of $12.18 \%$ was achieved indicating that the model worked accurately and reliably on every tested bearing.
\end{abstract}

Keywords: Fuzzy neural networks, Particle filtering, Prognostics and health management, Remaining useful life.

\section{Introduction}

Downtime caused by premature failures of mechanical components, driven by bearings or gearboxes, challenges almost industrial processes and increases the maintenance cost. In this context, the Prognostics and Health Management (PHM), as an approach to system life-cycle support, seeks to reduce or eliminate inspections and time-based maintenance [1]. Among the Architectures proposed for the PHM process, the Open System Architecture (OSA-PHM), which consists of seven layers ranging from data acquisition to the recommendation of mitigation actions, including in between detecting, identifying, isolating, predicting and managing faults and failures [2], Fig. 1. Enormous works have been focused on the first four layers of the OSAPHM architecture; sensing, signal processing, fault detection, and diagnostics, but there is still further to do.

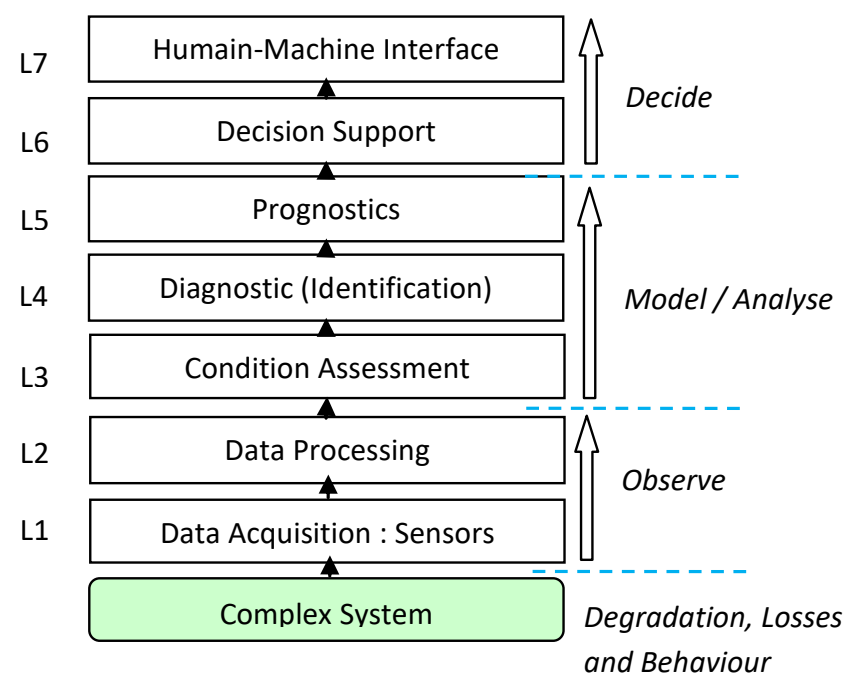

Figure. 1 OSA-CBM architecture 
The prognostic, which is the key process of the PHM, focuses mainly on predicting the RUL of a component/system with a confidence interval [2]. It can be achieved by using three main approaches namely model-based, data-driven and hybrid approaches [3]. Understanding pathological phenomena of failure and underlying physic laws that govern it is crucial to build an accurate degradation based model for the prognostic purpose. In the lack of this knowledge, data-driven techniques are preferred and bring a compromise between precision and complexity [4]. The strategies chosen by these techniques for RUL prediction: can be summarized in 1) finding a degradation indicator and predicting the trend until a threshold; 2) direct mapping from extracted features to RUL by regression [5]. Beneath the first category, degradation-based prognostic algorithms, called also projection algorithms, aim to track a prognostic feature, even sensed or pre-processed, reflecting the fault evolution through the entire component/system life; i.e. having a monotonic trend shape. In this framework, a lot of works have been dedicated to assess the RUL of the bearings. They are mainly categorized in two approaches, statistical approaches and artificial intelligent (AI) approaches [6-8]. The precision of these algorithms in predicting the RUL is tributary on the monotonicity and trendability of the prognostic features.

Implementing these methods in a classical way i.e. starting from feature extraction step of the acquired raw vibration signal, then evaluating the current health condition of a bearing through a health indicator and finally projecting the health indicator until it reaches a failure threshold rises some drawbacks that needed to be resolved. Theses drawbacks are summarised below:

- The identification of the suitable prognostic features of the available data left to expert analysis and engineering judgment, or typically identified through visual inspection are time consuming and tedious. These features can exhibit different degradation signatures at different levels of the degradation process. The trend of some features can occur right before the failure of the component, as shown in Fig. 2 and $7 b$ which hampers the accurate prediction of the RUL. Others are not monotonic or very sensitive to the measurement noise;

- Merging multiple features into a single health indicator which is then used to predict the RUL is common practice in prognostic works. This assumption of dimensionality fails at presenting the state of a bearing that can exhibit multiple failure modes [9].

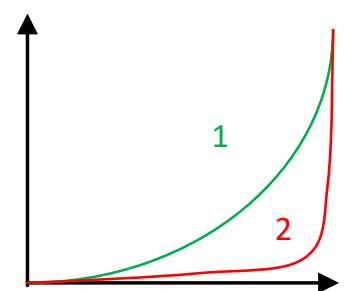

Figure. 2 Example of (1) high (2) low (monotonicity and trendability)

These methods typically require setting a failure threshold. A static threshold can be non-convenient, and an adaptive one is difficult to estimate [10].

To deal with all these issues is out of the scope of this study. Instead identifying a new feature with monotonic trend shape is a challenging problem and is our first goal. There is no such endeavour to tackle this problem, in our best knowledge, only few papers [11-15] deal with this issue. The common ground of all authors is to establish a set of shape quality metrics to evaluate the suitability of the features for the prognostic task.

For instance, the authors in [11] and [12] use a weighted sum of shape quality metrics namely trendability, monotonicity, and prognosability as fitness function and try to identify an optimal set of prognostic features based on their suitability for the prediction task from a population of features by merging them using genetic algorithm to extract the best of all together. These metrics are reported in section 2.2.3.

The authors in [13] instead, have proposed a fitness function based on the separability measure of successive time segments to assess the suitability of the extracted features for the RUL prediction.

The authors in [14] followed what was done in [11] and [12] and proposed a Genetic Programming (GP) as optimization approach which combines randomly mathematical operators, analytic functions, constants, and state variables to find out a mathematical expression of the advanced feature highly correlated with the fault growth using monotonicity as the only suitability metric. However, in addition to the hard understanding of the physical meaning of the identified feature, the difficulty, resides in determining failure threshold when different input features are concerned by the GP method.

Instead of dealing with an optimisation problem as the aforementioned authors do, [15] uses another approach based on the energy entropy, in which the normalized energy in the frequency spectrum is calculated over the cycles and the entropy is calculated as a trending feature. He claims that the 


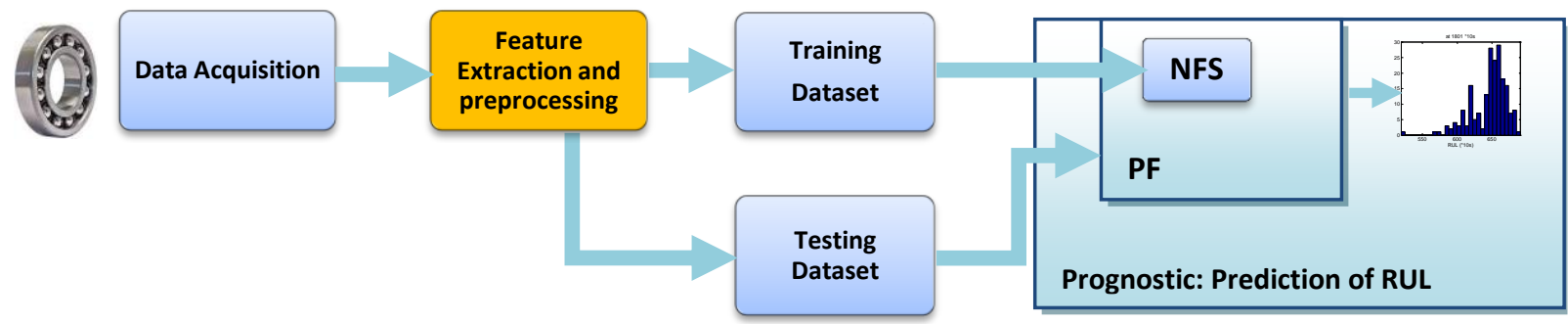

Figure. 3 Framework of the proposed RUL prediction approach

proposed energy entropy method shows smooth and constant decrease over the cycles which may represents the degree of fault progression.

Our approach to address this issue attempts firstly to acquire the trending shape by further preprocessing the extracted features transforming them to their cumulative form and secondly using Particle Filtering (PF) technique as a projecting algorithm to predict the RUL due to its capability to carry nonlinear systems in presence of non-Gaussian process/observation noise. But the use of $\mathrm{PF}$ technique raises another problem of induced errors since the system state evolution (i.e. bearing degradation feature in our case) usually integrated in $\mathrm{PF}$ as analytic expression even linear, logarithmic or exponential, kind of first order Markov Model, doesn't fit exactly the failure behavior. Again, to address this issue, we propose to use an offline trained model to be integrated later in PF. In our case, we propose a Neuro-Fuzzy System (NFS) predictor that fit perfectly the degradation process; Fig. 9.

This paper is organized in a way that it follows the flowchart of a typical health monitoring system. Starting from the data acquisition step in section 1, then the pre-processing step to identify the appropriate prognostic feature in section 2. The prediction of RUL is carried out using the adapted $\mathrm{PF}$ to the prognostic purpose with the integrated NFS as evolution state model in section 3 . The validation step ended our approach by the assessment of the results and the comparison with the previous works given in section 4 and 5 respectively. Finally, a conclusion is drawn in section 6 .

\section{Methodology}

The methodology followed here is that of the flowchart of Fig. 3.

The flowchart of the proposed method for bearing RUL prediction, illustrated in Fig. 3, includes two main phases: training phase and testing phase. The training phase conducted off-line aims to generate firstly a health indicator by further preprocessing the extracted feature from raw vibration signals acquired by the sensors and secondly to train the evolution model (NFS) used by particle filtering algorithm to fit the bearing degradation data. The testing phase is an on-line prediction that uses the generated model from the training phase to predict the RUL when a new test sample is available.

\subsection{Data acquisition step (L1)}

The choice of bearings and their condition monitoring to validate this study can be justified firstly, by the fact that these components are omnipresent in almost industrial processes using rotating elements and are subject to frequent failures. Secondly, when condition monitoring of bearings without altering their operation is considered, the vibrations is top ranked among the sensored data.

Note that the datasets were provided by FEMTO-ST Institute from an experimental test bench called PRONOSTIA.

\subsection{Data preprocessing step (L2)}

\subsubsection{Feature extraction}

The main goal of this step is to cartography the acquired data into a feature space related to the component health state using various signal processing techniques in time domain, frequency domain or time-frequency domain, [16]. For the PHM case study, none of the extracted features from raw vibration signal shows a monotonic trend shape.

\subsubsection{Feature preprocessing}

Motivated by the proposed model in [17] which states that the accumulated degradation for time interval $[0 \ldots \mathrm{t}]$ is proportional to the accumulated accelerations (vibrations). That means the influence of actual and high accelerations are more significant than the older and low accelerations. This is the case of mechanical components that do not undergo selfhealing like batteries. Therefore, transforming the extracted features into their cumulative form as 
health indicator using the cumulative sum (1) should well reflect the fault growth.

Note that the extracted features are presented as time series.

$$
c f(i)=\frac{\sum(\operatorname{data}(1: i))}{\sqrt{\left|\left(\sum(\operatorname{data}(1: i))\right)\right|}}
$$

where $\operatorname{data}(1: i)$ is the time series of any extracted feature up to time $i$.

$c f$ is the cumulative form of the time series data.

\subsubsection{Feature evaluation}

The evaluation of the suitability of the features for the prognostic task was made according to [11] using a set of suitability metrics namely monotonicity, trendability and prognosability, ranging from 0 to 1 for low and high suitability measure respectively. These metrics characterize the shape quality of the degradation curve.

Monotonocity stand for average fraction of positive and negative derivatives.

$$
\begin{aligned}
& \text { Monotonicity }= \\
& \qquad \operatorname{mean}\left(\left|\frac{\# \operatorname{pos}(d / d x)}{n-1}-\frac{\# n e g(d / d x)}{n-1}\right|\right)
\end{aligned}
$$

where $\# p o s(d / d x)$ is number of positive derivatives and $\# n e g(d / d x)$ is the number of negative derivatives

Prognosability is approximated as the variation of the final failure values for each degradation path divided by the mean range of the paths. It is exponentially weighted to provide the desired zero to one scale.

\section{Prognosability $=$}

$\exp \left(-\frac{s t d(\text { failurevalues })}{\text { mean }(\mid \text { failurevalue }- \text { startingvalue } \mid)}\right)$

The trendability is given by the smallest absolute correlation of the re-sampled prognostic feature according to the fraction of total lifecycle.

$$
\text { Trendability }=\min \left(\left|\operatorname{corrcoe} f_{i j}\right|\right)
$$

corrcoe $_{i j}$ are correlation coefficients of prognostic parameters

In addition to these metrics, we have also proposed the sum of the differential coefficients as another suitability measure. The differential coefficients are calculated after partitioning the degradation curve in equidistant time segments and using a linear regression for each part to calculate these coefficients.

\section{Remaining useful life prediction}

\subsection{Particle filtering technique adapted to prognostics purpose (L5)}

The Particle filtering is an interesting technique for sequential signal processing based on the concepts of Bayesian theory and Sequential Importance Sampling (SIS). It is particularly useful in dealing with nonlinear systems with the presence of non-Gaussian process/observation noise [18].

The posterior PDF is approximated each time point by a swarm of random samples, known as particles, with associated weights representing the discrete probability masses, drawn from the state evolution model [19-20].

The PF framework adapted for prognostic task is presented in Fig. 5 and it works as follow.

During the learning phase, we assume that the initial PDF $p\left(x_{0}\right)$ of the state is specified. So, the posterior PDF $p\left(x_{k} \mid z_{1: k}\right)$ is obtained recursively in two steps:

Prediction step: the particles are propagated using the state evolution model $p\left(x_{k} \mid x_{k-1}\right)$ (in our case the NFS model). We obtain a new PDF.

Update step: the weights $w_{k}^{i}$ are update on the light of measurement according to the calculated likelihood $p\left(z_{k} \mid x_{k}\right)$.

$$
w_{k}^{i} \approx w_{k-1}^{i} p\left(z_{k} \mid x_{k}^{i}\right)
$$

A re-sampling step is needed to avoid degeneration by eliminating the particles with lower weights.

At the end of this phase, when no measurement is available and the likelihood is no longer calculated; starts the prediction phase; only the state $x_{k}$ is propagated from one stage to another using the evolution model [21].

\subsection{Integration of NFS in particle filtering}

Analytic expressions even linear, exponential or logarithmic are often used to model the degradation 
process. These expressions are kind of first-order Markov models where the future system state $x_{k+1}$ depends only on the present state $x_{k}$ and a process noise $\omega_{k}$. As our degradation data exhibits a complex shape, approximating the degradation process by analytic expression induces errors. To address this issue, a Neuro-Fuzzy System (NFS) predictor was proposed to fit the degradation process. The proposed NFS is a fuzzy logic system combining the human-like reasoning style of fuzzy systems with the learning and connectionist structure of neural networks. He has the architecture of a single input-single output (SISO) system, Fig. 4,with five layers, wherein the signal is processed throw, namely input layer, Membership Function (MF) layer, rule layer, normalized layer and output layer, respectively [16] and [22].The system dynamics are handled by the process noise:

$$
\begin{aligned}
& x_{k+1}=\hat{x}_{k+1}+\omega_{k} \\
& \hat{x}_{k+1}=g_{k+1}\left(x_{k}\right)
\end{aligned}
$$

$g_{k+1}\left(x_{k}\right)$ is a nonlinear function used by the NFS and $\omega_{k}$ is the process noise.

\section{Experiment setup}

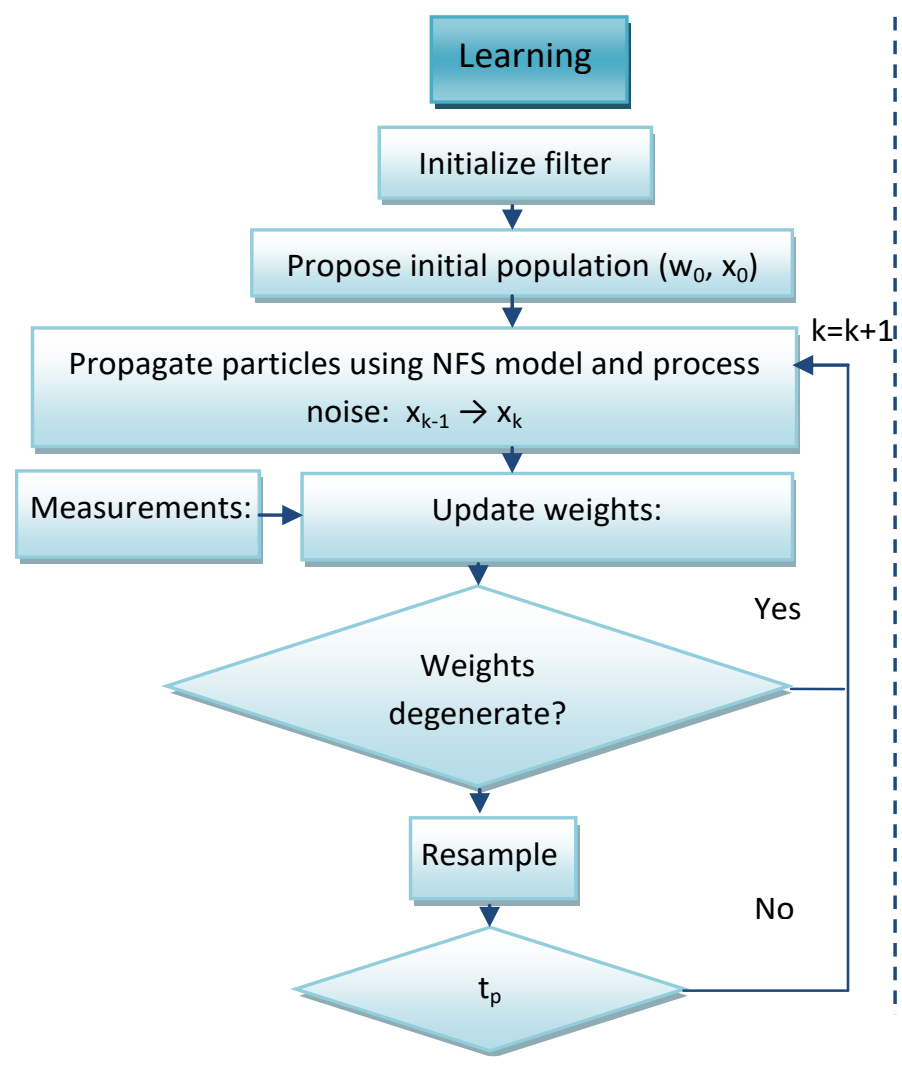

\subsection{PHM Case Study (Bearings Datasets of IEEE PHM Challenge 2012)}

The datasets were provided by FEMTO-ST Institute from an experimental platform called PRONOSTIA and are mainly composed of run-tofailure vibration signals related to ball bearings. The datasets were recorded at a sampling frequency of acceleration and temperature of $25.6 \mathrm{kHz}$ and $0.1 \mathrm{~Hz}$ respectively. The experiments were stopped when amplitude of the vibration signal reaches $20 \mathrm{~g}$ limit (g: gravity). Further details are given in [23].

$\operatorname{Ber}_{i-j}$ stands for the bearing number $j$ under the load condition $i$. Figs. 6 (a) and (b) show the raw vibration signals of the bearing $B e r_{1-1}$ on the horizontal and vertical axis respectively.

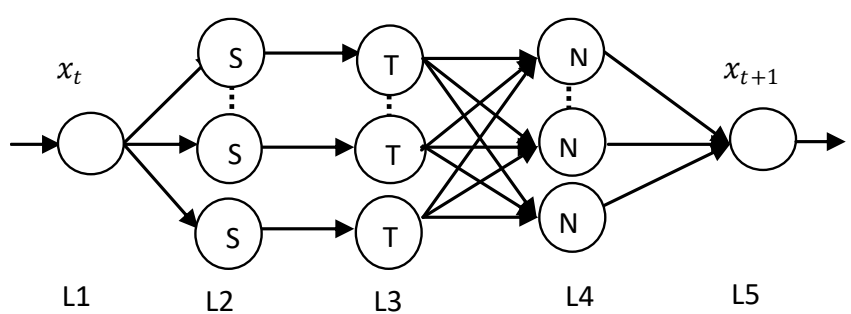

Figure. 4 SISO NFS predictor architecture

Figure. 5 Particle filter framework for prognostics 


\subsection{Feature extraction \& exploring}

Following the steps of the prognostic scheme and in addition to the statistical extracted features even dimensionally or dimensionless in time domain and the energies (defined as sum of the squares of amplitudes) over frequency bands centred on specific frequencies (e.g. rotating frequency and it harmonics and/or bearing defect frequencies) extracted using Fast Fourier Transform (FFT) which are taken as representative features in frequency domain, features in time-frequency domain are also considered, using the Daubichies wavelet of $4^{\text {th }}$ order D4 and $4^{\text {th }}$ decomposition level for the analysis of bearing vibration signals. We suppose that the $4^{\text {th }}$ decomposition level is enough to carry all the bearing defect frequencies and at least 3 of their harmonics according to [24].

Statistical features were performed on at different decomposition levels using detail coefficients. Fig. $7 \mathrm{a}$ and Fig $7 \mathrm{~b}$ are illustrating examples. These features still show a low trending and present variation right before failure time which limits their prognostic capabilities as mentioned before.

Instead, the extracted feature transformed to its cumulative form as health index feature, Fig. 8, exhibits a smoothed and trended shape and appears to be well adapted to the RUL prediction. This claim is substantiated by the results of the suitability comparison between the extracted and the preprocessed features, presented in Table 1 which show clearly that the pre-processed feature outperform the extracted one. The comparison was made using the suitability metrics according to [11] namely monotonicity, trendability, prognosability and the sum of the differential coefficients as another proposed measure of suitability. The comparison results were done on the fitted version of the extracted feature because these metrics are greatly affected by rapid fluctuations. The $11^{\text {th }}$ order polynomial fitting was used in our case to handle the global trend and local variations.

For the sake of emphasizing the comparison between the two normalized features of Fig. 8b (i.e. the extracted feature compared to the pre-processed one), we present the results of the RUL predictions in Fig. 11. These results are very close to the true RUL. An average error percentage of $17.76 \%$ was achieved instead of $81.87 \%$.

The given threshold for the training vibration dataset was $20 \mathrm{~g}$ correspond to $37,5 \mathrm{~g}$ on the cumulative form which was kept for the test phase.
Table 1. Results of the suitability comparison of the extracted feature vs its cumulative form

\begin{tabular}{|l|l|l|}
\hline & $\begin{array}{l}\text { normalized } \\
\text { cumulative } \\
\text { feature }\end{array}$ & $\begin{array}{l}\text { normalized } \\
\text { Wrms_5 }\end{array}$ \\
\hline Monotonocity & 1 & 0.8064 \\
\hline Trenddability & 0.9934 & 0.8799 \\
\hline Prognosability & 1 & 1 \\
\hline $\begin{array}{l}\text { Sum of differential } \\
\text { coefficients (proposed) }\end{array}$ & 0.0328 & 0.0138 \\
\hline
\end{tabular}

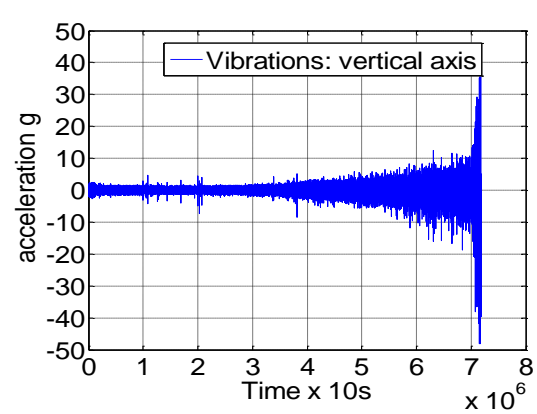

(a)

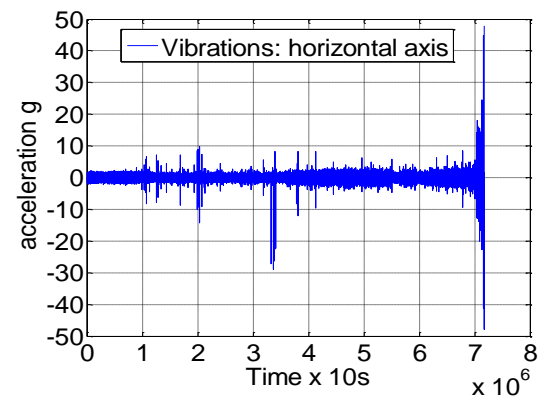

(b)

Figure. 6 Vibration signals of the bearing $\mathrm{Ber}_{1-1}$ : (a) horizontal and (b) vertical axis

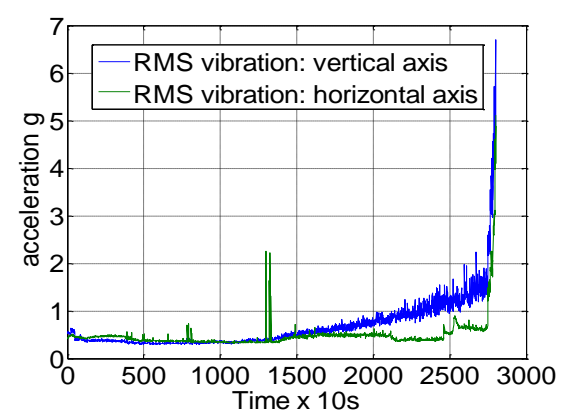

(a)

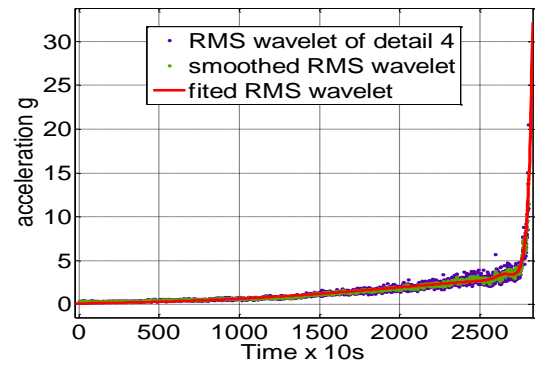

(b)

Figure. 7 (a) RMS vibration features of bearing Ber $_{1-1}$ and (b) RMS of wavelet detail coefficients of level 4 of bearing Ber $_{1-1}$ with its fitting curve (red) 


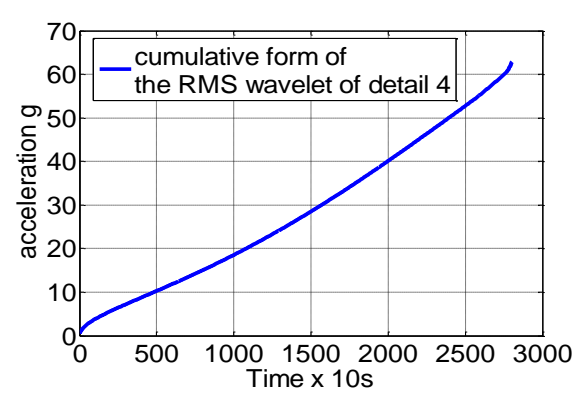

(a)

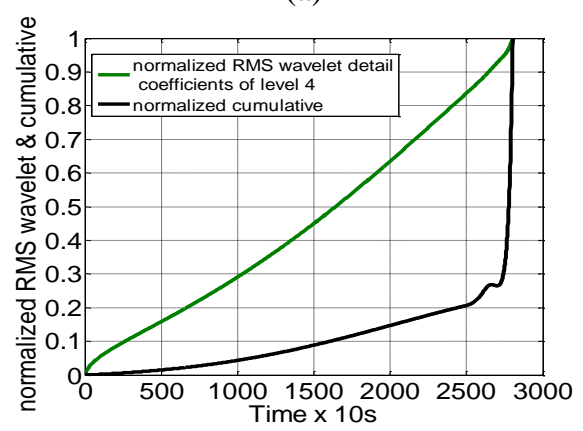

(b)

Figure. 8 (a) Identified feature for the bearing Ber $_{1-1}$ and (b) Normalized versions of the identified feature and the fitted curve of RMS wavelet detail coefficients of level 4

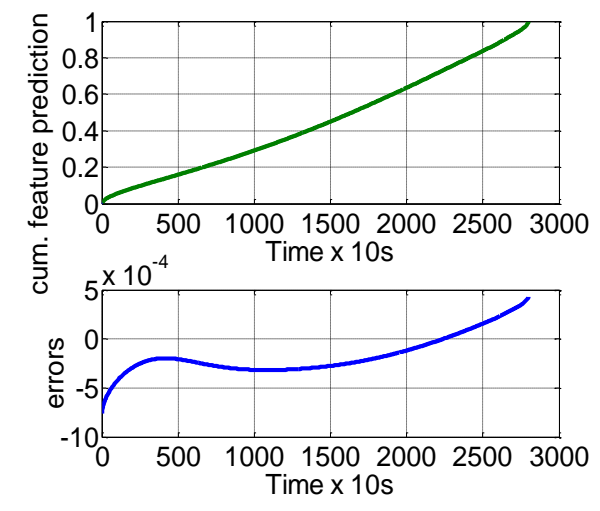

Figure. 9 Results of NFS evolution mode

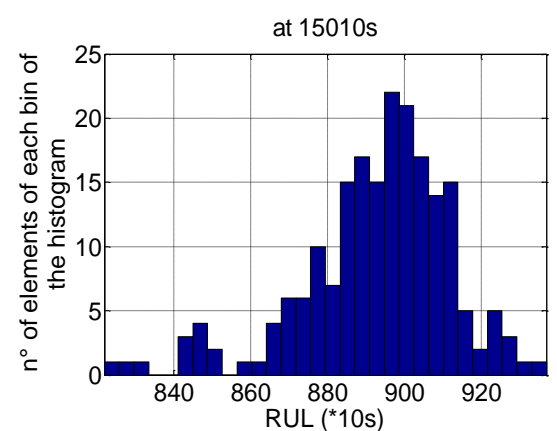

Figure. 10 Example PDF RUL prediction at 15010s of Ber $_{1-7}$

\section{Comparison to previous works}

The percentage error of RUL prediction is calculated according to [23] as:

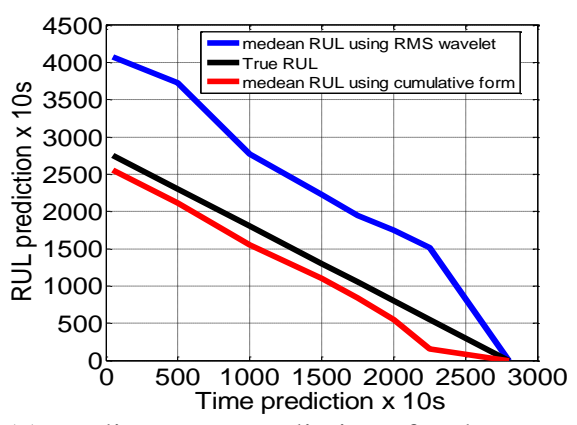

Figure. 11 Median RUL predictions for the wavelet RMS

(blue) and its cumulative form (red) - Ber $_{1-3}$

$$
\% E R=100 \times \frac{\operatorname{actRUL}-\widehat{R U L}}{\operatorname{actRUL}}
$$

where actRUL is the actual RUL and $\widehat{R U L}$ the predicted one. A negative value indicates a late prediction, while a positive value indicates an early prediction.

To benchmark the proposed method, we present in Tables 2 and 3 the results of some published works for bearing RUL prediction using the same PRONOSTIA datasets. The strategies taken in almost works can be summarized in two main points: 1) identifying a health indicator then extrapolating it until it reaches a predefined threshold; 2) using regression to map directly the health indicator to RUL prediction.

The prediction results of the RUL are depicted in Table 2. For each testing bearing, the prediction is made at the end of test data. The method proposed by [26], winner of the IEEE PHM 2012 Prognostic Challenge, predicts the RUL using frequency analysis-based anomaly detection, degradation feature extrapolation, and survival time ratios. [26] extracted a prognostic feature by taking averaging of five highest absolute acceleration values measured in each observation, realized degradation state recognition of bearings by anomaly detection when there was a change in the frequency of the peak vibration in the frequency spectrum and estimated RUL based on making comparisons on durations of degradation states between the training and the test bearings. However, the method has the disadvantage of defining the anomaly detection time point based on subjective criteria used to calculate the bearing survival time ratios. Instead of looking for an overall regression model for RUL prediction, [27] proposes a RUL prediction framework based on multiple health state assessment that divides the entire bearing life into several health states where a local regression model can be built individually based on multiple health state assessment and adopt SVM as 
Table 2. Performance comparisons of the proposed method with related research on the PRONOSTIA dataset

\begin{tabular}{|l|c|c|c|c|c|c|c|c|c|c|}
\hline & Current & actual & \multicolumn{10}{|c|}{ RUL predicted by } \\
\cline { 8 - 14 } & point (s) & RUL & {$[26]$} & {$[27]$} & {$[28]$} & {$[29]$} & {$[30]$} & {$[10]$} & L $_{10}$ & Proposed method \\
\hline Bearing 1_3 & 18010 & 5730 & 3610 & 5842 & 5790 & 3250 & 5750 & 5670 & 15,323 & 6520 \\
\hline Bearing1_4 & 11380 & 339 & 68 & 1109 & 410 & 110 & 320 & 270 & 21,953 & - \\
\hline Bearing1_5 & 23010 & 1610 & 1465 & - & 6090 & 1980 & 0 & 1430 & 10323 & 1210 \\
\hline Bearing1_6 & 23010 & 1460 & 1533 & - & 1460 & 1150 & 1050 & 950 & 10323 & 1475 \\
\hline Bearing1_7 & 15010 & 7570 & 7721 & - & 7560 & 6220 & 6090 & 5360 & 18323 & 8275 \\
\hline
\end{tabular}

Table 3. Percentage error $\% E R$

\begin{tabular}{|c|c|c|c|c|c|c|c|c|}
\hline & \multicolumn{8}{|c|}{$\% E R$} \\
\hline & [26] & [27] & [28] & [29] & [30] & [10] & $\mathrm{L}_{10}$ & Proposed method \\
\hline Bearing 1_3 & 37 & -2 & $-1,04$ & 43,28 & $-0,35$ & 1,05 & -167 & $-13,78$ \\
\hline Bearing1_4 & 80 & 62 & $-20,94$ & 67,55 & 5,60 & 20,35 & -660 & - \\
\hline Bearing1_5 & 9 & - & $-278,26$ & $-22,98$ & 100 & 11,18 & -541 & 24,64 \\
\hline Bearing1_6 & -5 & - & 19,18 & 21,23 & 28,08 & 34,93 & -607 & -1 \\
\hline $\begin{array}{c}\text { Bearing1_7 } \\
\text { mean }\end{array}$ & $\begin{array}{c}-2 \\
26,6\end{array}$ & - & $\begin{array}{l}-7,13 \\
65,31\end{array}$ & $\begin{array}{l}17,83 \\
\mathbf{3 4 , 5 7}\end{array}$ & $\begin{array}{l}-19,55 \\
\mathbf{3 0 , 8 6}\end{array}$ & $\begin{array}{l}29,19 \\
\mathbf{1 9 , 3 4}\end{array}$ & $\begin{array}{l}-142 \\
\mathbf{4 2 3}, \mathbf{4}\end{array}$ & $\begin{array}{c}9,31 \\
\mathbf{1 2 , 1 8}\end{array}$ \\
\hline
\end{tabular}

the technique to implement both health state assessment and local RUL prediction. Another work was published by [28]. They used wavelet packetEMD for feature extraction and SOM for constructing the HI. The method shows improved errors over previous studies, but requires the extraction of approximately one hundred features to estimate bearing performance. [29] published an RNN-based RUL prediction method constructed by selecting and fusing multiple features extracted from the time, frequency, and time-frequency domains. To improve the accuracy of RUL prediction, a deep learning approach was adopted. This method demonstrated its superiority over SOM-based HI. [30], proposed a new HI (i.e.,WMQE) to predict the RUL of bearings. WMQE was constructed by fusing a select few weighted features based on correlation clustering among the 28 features extracted from the bearings. The study showed the best performance among existing studies. A most recent study published by [10] use image features to construct a HI. The CNN model was used as a regression model to estimate the HI between 0 and 1 . The estimated CWTCNN-HI was used for the RUL prediction. A deep learning approach was adopted to train the CWTCNN-HI model.

The proposed method took the first strategy, i.e. identifying a health indicator then extrapolating it until it reaches a predefined threshold, and was based essentially on transforming the extracted features into their cumulative form to construct the HI then using the PF technique as extrapolating tool. The results depicted in Table 3 show overall low percentage errors, A mean percentage error of $12.18 \%$ was achieved, indicating that the model worked accurately and reliably on every tested bearing except for the bearing Ber $_{1-4}$ where major deviation was found and which was then discarded. Compared to the aforementioned works, the proposed method would be a good compromise if the goal is to decrease the computational requirements with an error that does not increase too much.

\section{Conclusion}

As found in the several case studies including ours, traditional extracted features have shown undesirable behaviors such as fluctuation, nonmonotonicity and abrupt increase at the end of the component's life cycle which impair the prediction accuracy.

The problem of enhancing the RUL was tackled with a new way; i.e. we have focusing on shifting the degradation behavior from an abrupt change at the end of the component's life cycle to a monotonic and smoothed trend shape through the entire component's life cycle reflecting the fault growth rather than dealing with projection algorithms in attempt to improve the prediction accuracy or seeking for extracted features which were intrinsically trendables as many works do.

The first goal of the proposed approach was mainly achieved by further pre-processing the extracted features transforming them into their cumulative form as health indicator. The identified health indicator exhibits, this time, a monotonic trend that clearly reflects the fault evolution of the component, compared to its original form. The assessment of the identified health indicator qualities was performed by a set of proposed and established suitability metrics which show clearly 
the superiority of the identified features over the extracted one.

As a projection tool, we have adopted the $\mathrm{PF}$ technique for the prediction of the RUL due to its capability to carry nonlinear systems in presence of non-Gaussian process/observation noise with the NFS predictor that fit perfectly the degradation process to simulate the state model evolution.

The proposed approach was validated through a set of acquired bearing run to failure datasets. The results of the RUL predictions are very convincing and quite close to the true ones. A mean percentage error of $12.18 \%$ was achieved, indicating that the model worked accurately and reliably on every tested bearing.

The strong point of this method lies in its simplicity and simplicity implies less computing compared to other studies that invest in accuracy at the expense of computing time. This study makes a good compromise between accuracy and less computing.

The challenge remaining for the future work is to reduce the uncertainty of the RUL prediction.

\section{References}

[1] Z. S. Chen, Y. M. Yang, and H. Zheng, "A Technical Framework and Roadmap of Embedded Diagnostics and Prognostics for Complex Mechanical Systems in Prognostics and Health Management Systems", IEEE Transactions on Reliability, Vol. 61, No. 2, pp. 314-322, 2012.

[2] DIN EN 13306: 2010-12, "Maintenance terminology", (2010).

[3] A. K. Jardine, D. Lin, and D. Banjevic, "A Review on Machinery Diagnostics and Prognostics Implementing Condition-Based Maintenance", Journal of Mechanical Systems and Signal Processing, Vol. 20, No. 7, pp. 1483-1510, 2006.

[4] M. Baptista, I. P. Medeiros, J. P. Malere, C. Nascimento, H. Prendinger, and E. M. P. Henriques, "Comparative Case Study of Life Usage and Data-Driven Prognostics Techniques Using Aircraft Fault Messages", Computers in Industry, Vol. 86, pp. 1-14, 2017

[5] B. Huang, Y. Di, C. Jin, and J. Lee, "Review Of Data-Driven Prognostics And Health Management Techniques: Lessons Learned From PHM Data Challenge Competitions", In: Proc. of Machine Failure Prevention Technology, pp. 1-17, 2017.

[6] B. Zhang, C. Deng, and Y. Zhang "A Hybrid Feature Selection and Health Indicator
Construction Scheme for Delay-Time-Based Degradation Modeling of Rolling Element Bearings", IOP Conference Series: Materials Science and Engineering, Vol. 339, 2018.

[7] Y. Peng, M. Dong, and M. J. Zuo, "Current Status of Machine Prognostics in ConditionBased Maintenance: a Review", International Journal of Advanced Manufacturing Technology, Vol. 50, No.1-4, pp. 297-313, 2010.

[8] J. Z. Sikorska, M. Hodkiewicz, and L. Ma, "Prognostic Modeling Options for Remaining Useful Life Estimation by Industry", Journal of Mechanical Systems and Signal Processing, Vol. 26, No. 5, pp. 1803 - 1836, 2011.

[9] A. Z. Hinchi and M. Tkiouat, "Rolling Element Bearing Remaining Useful Life Estimation Based on a Convolutional LongShort-Term Memory Network", Procedia Computer Science, Vol. 127, pp. 123-132, 2018.

[10] Y. Yoo and J.G. Baek, "A Novel Image Feature for the Remaining Useful Lifetime Prediction of Bearings Based on Continuous Wavelet Transform and Convolutional Neural Network", Applied Sciences, Vol. 8, No. 7, pp. 1-17, 2018.

[11] J. Coble and J. W. Hines, "Identifying Optimal Prognostic Parameters from Data: A Genetic Algorithms Approach", Annual Conference of the Prognostics and Health Management Society, 2009.

[12] J. Coble, "Merging Data Sources to Predict Remaining Useful Life - An Automated Method to Identify Prognostic Parameters", $\mathrm{PhD}$, University of Tennessee 2010. [Online]. http://trace.tennessee.edu/utk_graddiss/683.

[13] F. Camci, K. Medjaher, N. Zerhouni, and P. Nectoux, "Feature Evaluation for Effective Bearing Prognostics", Quality and Reliability Engineering International, pp. 1-15, 2012.

[14] L. Liao. "Discovering Prognostic Features Using Genetic Programming in Remaining Useful Life Prediction", IEEE Transactions on Industrial Electronics, Vol. 61, No. 5, pp. 2464-2472, 2014.

[15] S. G. Kim, S. Park, D. An, J. W. Kim, J. Han, N. H. Kim, and J. H. Choi, "A New Prognostics Approach for Bearing Based on Entropy Decrease and Comparison with Existing Methods", Annual Conference of the Prognostics and Health Management Society, 2016.

[16] T. Boukra, "Identifying New Prognostic Features for Remaining Useful Life Prediction Using Particle Filtering and Neuro-Fuzzy 
System Predictor", In: Proc. of IEEE 15th International Conference on Environment and Electrical Engineering, pp. 1533-1538, June 2015.

[17] S. Porotsky and Z. Bluvband, "Remaining Useful Life Estimation for Systems with NonTrendability Behaviour", In: Proc. of IEEE Conference on Prognostics and Health Management, 2012.

[18] M. S. Arulampalam, S. Maskell, N. Gordon, and T. Clapp, "A Tutorial on Particle Filters for Online Nonlinear/Non-Gaussian Bayesian Tracking", IEEE Transactions on Signal Processing, Vol. 50, pp. 174-188, 2002.

[19] S. Yin and X. Zhu, "Intelligent Particle Filter and Its Application to Fault Detection of Nonlinear System", IEEE Transactions on Industrial Electronics, Vol. 62, No. 6, pp: 3852-3861, 2015.

[20] M. Orchard and G.A. Vachtsevanos, "Particle Filtering Approach for On-Line Fault Diagnosis and Failure Prognosis", Transactions of the Institute of Measurement and Control, Vol. 31, No. 3-4, pp. 221-246, 2009.

[21] M. Jouin, R. Gouriveau, D. Hissel, M. C. Péra and N. Zerhouni, "Joint Particle Filters Prognostics for Proton Exchange Membrane Fuel Cell Power Prediction at Constant Current Solicitation", IEEE Transactions on Reliability, Vol. 65, No. 1, pp. 336-349, 2016.

[22] C. Chen, B. Zhang, and G. Vachtsevanos "Prediction of Machine Health Condition Using Neuro-Fuzzy and Bayesian Algorithms", IEEE Transaction on Instrumentation and Measurement, Vol. 61, No. 2, pp. 297-306, 2012.

[23] IEEE PHM 2012 prognostic challenge [online] www.femto-st.fr/f/d/ieeephm2012-challengedetails.pdf.

[24] D. Tobon-Mejia, K. Medjaher, N. Zerhouni, and G. Tripot, "A Data Driven Failure Prognostics Method Based on Mixture of Gaussians Hidden Markov Models", IEEE Transactions on Reliability, Vol. 61, No. 2, pp. 491-503, 2012.

[25] A. Saxena, J. Celaya, E. Balaban, K. Goebel, B. Saha, S. Saha, and M. Schwabacher, "Metrics for Evaluating Performance of Prognostic Techniques", In: Proc. of the International Conference on Prognostics and Health Management, pp. 1-17, 2008.

[26] E. Sutrisno, H. Oh, A.S.S. Vasan, and M. Pecht, "Estimation of Remaining Useful Life of Ball Bearings Using Data Driven Methodologies",
In: Proc. of IEEE Conference on Prognostics and Health Management, pp. 1-7, 2012.

[27] Z. Liu, M. J Zuo, and Y. Qin, "Remaining Useful Life Prediction of Rolling Element Bearings Based on Health State Assessment", Journal of Mechanical Engineering Science, pp. $1-17,2015$.

[28] S. Hong, Z. Zhou, E. Zio, and K. Hong, "Condition Assessment for the Performance Degradation of Bearing Based on a Combinatorial Feature Extraction Method", Digital Signal Processing, Vol. 27, pp. 159166, 2014.

[29] L. Guo, N. Li, F. Jia, Y. Lei, and J. A Lin, "Recurrent Neural Network Based Health Indicator for Remaining Useful Life Prediction of Bearings", Neuro-Computing, Vol. 240, pp. 98-109, 2017.

[30] Y. Lei, N. Li, S. Gontarz, J. Lin, S. Radkowski, and J. Dybala, "A Model-Based Method for Remaining Useful Life Prediction of Machinery", IEEE Transaction Reliability, Vol. 65, pp. 1314-1326, 2016. 